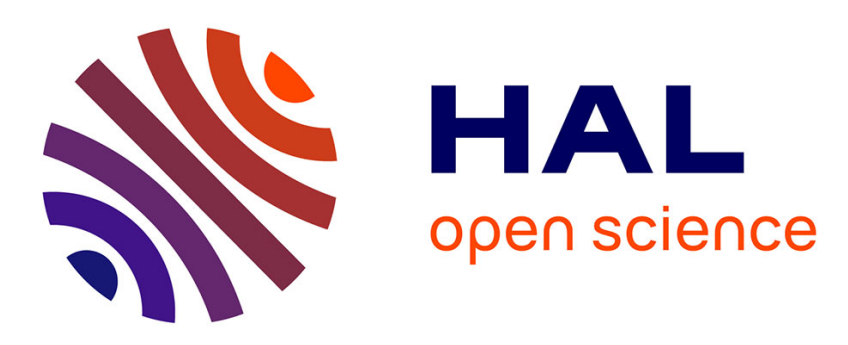

\title{
Current-induced magnetization reversal in terms of power dissipation
}

\author{
J. Cucchiara, Eric E. Fullerton, A. D. Kent, J. Z. Sun, Y. Henry, Stéphane \\ Mangin
}

\section{> To cite this version:}

J. Cucchiara, Eric E. Fullerton, A. D. Kent, J. Z. Sun, Y. Henry, et al.. Current-induced magnetization reversal in terms of power dissipation. Physical Review B: Condensed Matter and Materials Physics (1998-2015), 2011, 84 (10), pp.100405. 10.1103/PhysRevB.84.100405 . hal-01624060

\section{HAL Id: hal-01624060 \\ https://hal.univ-lorraine.fr/hal-01624060}

Submitted on 2 Nov 2017

HAL is a multi-disciplinary open access archive for the deposit and dissemination of scientific research documents, whether they are published or not. The documents may come from teaching and research institutions in France or abroad, or from public or private research centers.
L'archive ouverte pluridisciplinaire $\mathbf{H A L}$, est destinée au dépôt et à la diffusion de documents scientifiques de niveau recherche, publiés ou non, émanant des établissements d'enseignement et de recherche français ou étrangers, des laboratoires publics ou privés. 
PHYSICAL REVIEW B 84, 100405(R) (2011)

\title{
Current-induced magnetization reversal in terms of power dissipation
}

\author{
J. Cucchiara \\ Institut Jean Lamour, CNRS, Nancy Université, UPV Metz, Vandoeuvre lès Nancy, France
}

Eric E. Fullerton

Center for Magnetic Recording Research, University of California, San Diego, California 92093, USA

A. D. Kent

Department of Physics, New York University, New York, New York 10003, USA

J. Z. Sun

IBM T. J. Watson Research Center, P.O. Box 218, Yorktown Heights, New York 10598, USA

Y. Henry
Institut de Physique et Chimie des Matériaux de Strasbourg, CNRS, UDS, Strasbourg, France

\section{S. Mangin*}

Institut Jean Lamour, CNRS, Nancy Université, UPV Metz, Vandoeuvre lès Nancy, France

(Received 18 April 2011; revised manuscript received 7 June 2011; published 21 September 2011)

\begin{abstract}
Magnetization excitation and reversal induced by spin-transfer torques is described in terms of power received or dissipated in a macrospin system. This approach provides a clear and intuitive understanding of the effect of both applied magnetic fields and injected spin-polarized currents on magnetization reversal. It is illustrated by solving the case of magnetization reversal in a nanopillar spin valve with perpendicular magnetizations although the approach can be applied more generally. The appearance of critical currents below which spin-transfer torque is no longer efficient is explained by a break in the uniaxial symmetry of such structures.
\end{abstract}

PACS number(s): 75.60.Jk, 72.25.Ba, 75.60.Ch, 85.75.-d

What is now known as the Landau-Lifshitz-Gilbert (LLG) equation $^{1}$ was introduced in 1935 to model the precession motion of magnetization in a solid considering an effective magnetic field $\boldsymbol{H}_{\text {eff }}$ and a damping coefficient $\alpha$. It quickly became an important tool for modeling magnetization reversal even in complex system. On the other hand, magnetization reversal of a single domain (macrospin) can also be described by the Stoner-Wohlfarth model, ${ }^{2}$ which considers the magnet's energy and transitions between metastable and stable magnetic states. This latter description often provides a clear physical picture of the magnetic behavior.

Since the prediction of spin-transfer phenomena by Berger $^{3}$ and Slonczewski, ${ }^{4}$ magnetization reversal driven by spin-polarized current injection has been modeled primarily by adding the "Slonczewski" spin-transfer torque term to the LLG equation. This Landau-Lifshitz-Gilbert-Slonczewski (LLGS) equation describes current-induced magnetization dynamics very accurately and is successfully used in micromagnetic simulations. ${ }^{5}$ Current induced magnetization dynamic on short time scales in nanopillar has been reported. ${ }^{6,7}$ The StonerWohlfarth model is generally not applied on the basis that spin torque is a non-energy-conserving torque. In this Rapid Communication, we show how to include spin-transfer torque into the Stoner-Wohlfarth model. We present a description of magnetization reversal in the spirit of the Stoner-Wohlfarth energetic approach by considering power dissipation to reflect the roles of damping and spin-transfer torque on the system. In the following, the method is first described and then illustrated for current-induced magnetization reversal in the case of nanopillar spin valves with perpendicular magnetic anisotropy.
Qualitative description. In the Stoner-Wohlfarth model, one considers that stable equilibrium states correspond to minima in the magnetic energy landscape, i.e., that the magnetic system will dissipate energy to the environment and end up in a minimum of energy. The total power $P_{\mathrm{t}}$ received from the outside is then defined as negative. With the addition of the spin-transfer torque, magnetization can also receive energy which, if sufficient, can overcome dissipation. Thus, $P_{\mathrm{t}}$ may be either positive or negative. As a result, others states are now stabilized: The basic idea is that minima are stabilized by a negative power and maxima by a positive power. ${ }^{8}$ For the simplest energy landscape consisting of two equilibrium states, one being an energy minimum and the other one a maximum, four scenarios need to be investigated, as shown schematically in Fig. 1. In the case of pure dissipation $\left(P_{\mathrm{t}}<0\right)$ only the minimum is stable [Fig. 1(a)]. If the system receives energy $\left(P_{\mathrm{t}}>0\right)$ for both equilibrium states then the stable equilibrium position is the maximum [Fig. 1(b)]. However, if the power received depends on the equilibrium states of the system, either both the minimum and the maximum may be stable [Fig. 1(c)] or both are unstable [Fig. 1(d)]. In this latter case, the power received must cancel somewhere between the two equilibrium states and the magnetization is in a dynamic equilibrium state.

Therefore, to study the evolution of magnetic states requires both (1) the magnetic energy landscape, which provides the energy minima and maxima, i.e., the equilibrium positions and (2) the power received by the system in the vicinity of the equilibrium positions whose sign determines the stability of these positions. Of course, the power received at the exact equilibrium positions is always equal to zero so in 


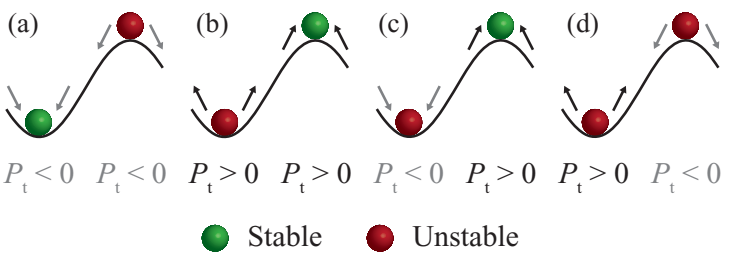

FIG. 1. (Color online) Schemes of the four different stability configurations available in a simple energy landscape composed of one minimum and one maximum. For each energy minimum and maximum position the power $P_{\mathrm{t}}$ received from the outside may be either negative or positive. The arrows represent the direction of the torque felt by the system as it is moved away from its equilibrium position.

the following, the power will refer as the power received by the system when its magnetization is slightly shifted from its equilibrium position. Note that calculating the power is equivalent to a stability analysis. ${ }^{9}$

Quantitative description. The magnetic system is characterized in the macrospin approach by its magnetic energy $E(\boldsymbol{m}, \boldsymbol{H})$, where $\boldsymbol{m}$ is a unit vector in the direction of the magnetization and $\boldsymbol{H}$ the applied magnetic field. This energy takes into account all the conservative interactions acting on the system.

The effective magnetic field can be derived from the magnetic energy $\boldsymbol{H}_{\text {eff }}=-\left(\frac{1}{\mu_{0} M_{\mathrm{s}} V}\right) \frac{\partial E}{\partial \boldsymbol{m}}$, with $\mu_{0}$ the permeability of free space, $M_{\mathrm{S}}$ the saturation magnetization, and $V$ the volume of the magnet. The dynamics of the system is then captured by three torques acting on the magnetization: the field torque $\boldsymbol{\Gamma}_{\mathbf{f}}=-\gamma \mu_{0} \boldsymbol{m} \times \boldsymbol{H}_{\text {eff }}$, the damping torque $\boldsymbol{\Gamma}_{\mathbf{d}}=-\alpha \gamma \mu_{0} \boldsymbol{m} \times\left(\boldsymbol{m} \times \boldsymbol{H}_{\text {eff }}\right)$, and the spin-transfer torque $\boldsymbol{\Gamma}_{\mathrm{s}}=-\beta \boldsymbol{I} \boldsymbol{m} \times(\boldsymbol{m} \times \boldsymbol{u})$, where $\gamma$ is gyromagnetic factor and $I$ is the intensity of the current. $\beta$ is given by $\frac{\hbar \gamma \mathcal{G}(P, \boldsymbol{m} \cdot \boldsymbol{u})}{2 e M_{\mathrm{s}} V}$, where $\hbar$ is the reduced Planck constant and $\mathcal{G}(P, \boldsymbol{m} \cdot \boldsymbol{u})$ is the efficiency function derived by Slonczewski ${ }^{4}$ with $P$ the current polarization, $\boldsymbol{u}$ the unit vector in the direction of the current polarization, and $e$ the elementary charge. The field torque is responsible for the precession of the magnetization around the direction of the effective magnetic field and is a conservative torque. It gives the rotation vector of the system $\boldsymbol{\Omega}=\gamma \mu_{0} \boldsymbol{H}_{\text {eff }}$. The damping and the spin-transfer torques are nonconservative. The power received by the magnetization from these torques are respectively given by $P_{\mathrm{d}}=\boldsymbol{\Omega} \cdot \boldsymbol{\Gamma}_{\mathbf{d}}$ and by $P_{\mathrm{s}}=\boldsymbol{\Omega} \cdot \boldsymbol{\Gamma}_{\mathrm{s}}$. The total power received by the magnetization is given by $P_{\mathrm{t}}=P_{\mathrm{d}}+P_{\mathrm{s}}$.

Therefore, computing $\frac{\partial E}{\partial \boldsymbol{m}}=\mathbf{0}$ to get the equilibrium positions and the sign of $P_{\mathrm{t}}$ to determine their stability for any applied magnetic field and current, one can build the state diagram of the system. A state diagram presents the available stable magnetic states depending on the applied magnetic field and the injected current.

Application. Let us now consider a spin-valve structure in the current-perpendicular-to-plane geometry at zero temperature. The magnetization of the hard layer is fixed in the out-of-plane direction and so is the polarization of the injected electrons. The magnetic field is also applied in this direction. The injected current is defined as positive when it flows from the free layer to the hard layer. The magnetic field is defined as positive when it is applied parallel to the magnetization of the hard layer. The free layer is described by a macrospin with uniaxial anisotropy. Its easy anisotropy axis makes an angle $\lambda$ with the perpendicular direction. Note that if $\lambda=0$ the system has uniaxial symmetry. To break this symmetry one can choose to tilt the anisotropy axis $(\lambda \neq 0)$.

For $\lambda=0$ previous stability analysis of the LLGS equation showed that the switching fields of the free layer are linear with the injected current. ${ }^{10,11}$ As a consequence, its state diagram is similar to the one presented in the inset of Fig. 4. The following will show that the power dissipation approach is in perfect agreement with this description. However, experimental results exhibit large deviations from this linear behavior especially around the zero current switching field. ${ }^{12,13}$ Indeed, in those areas the switching fields are not or only weakly affected by the spin-polarized current. Using the power dissipation approach we will attribute this deviation to breaking of the uniaxial symmetry.

The magnetic energy of the free layer is given by

$$
E(\theta, \boldsymbol{H})=K V \sin ^{2}(\theta-\lambda)-\mu_{0} M_{\mathrm{s}} V \boldsymbol{m} \cdot \boldsymbol{H},
$$

where $\theta$ is the angle between the magnetizations of the hard and free layers and $K$ is the anisotropy constant. One can define the anisotropy field $H_{\mathrm{k}}=\frac{2 K}{\mu_{0} M_{\mathrm{s}}}$. The effective magnetic field derived from this energy has an in-plane $\left(H_{\text {eff }}^{\text {in }}\right)$ and an outof-plane $\left(H_{\mathrm{eff}}^{\text {out }}\right)$ component. The powers generated by the nonconservative damping and spin-transfer torques are given by

$$
\begin{gathered}
P_{\mathrm{d}}=-\alpha \gamma_{0} \mu_{0} M_{\mathrm{s}} V\left(H_{\mathrm{eff}}^{\text {in }} \cos \theta-H_{\mathrm{eff}}^{\text {out }} \sin \theta\right)^{2}, \\
P_{\mathrm{s}}=\mu_{0} M_{\mathrm{s}} V \beta\left(H_{\mathrm{eff}}^{\text {in }} \sin \theta \cos \theta-H_{\mathrm{eff}}^{\text {out }} \sin ^{2} \theta\right) I .
\end{gathered}
$$

Note that $P_{\mathrm{d}}$ is always negative whereas $P_{\mathrm{s}}$ is either positive or negative depending on the sign of the current and on the orientation of the effective field. The calculated power is the mean value of the total power received by the magnetization when it is tilted away from its equilibrium position by an angle $\pm \delta \theta$ with $\delta \theta=0.05^{\circ}$.

For clarity purposes, let us first study the system with the uniaxial symmetry $\left(\lambda=0 \Rightarrow H_{\text {eff }}^{\text {in }}=0\right)$. Figure $2($ a) presents the evolution of the magnetic energy of the free layer as a function of $\theta$ for different applied magnetic fields. In this uniaxial case, the zero current switching field $H_{\mathrm{sw}}^{0}$ is equal to $H_{\mathrm{k}}$. Four different equilibrium states appear for different ranges of magnetic fields: two minima called $E_{\min }^{-}(H<$ $\left.H_{\mathrm{sw}}^{0}\right)$ and $E_{\mathrm{min}}^{+}\left(H>-H_{\mathrm{sw}}^{0}\right)$ and two maxima called $E_{\mathrm{max}}^{-}$ $\left(H<H_{\mathrm{sw}}^{0}\right)$ and $E_{\mathrm{max}}^{+}\left(H>-H_{\mathrm{sw}}^{0}\right)$. The magnetization angular positions corresponding to these states (respectively noted $\theta_{\min }^{-}, \theta_{\min }^{+}, \theta_{\max }^{-}$, and $\theta_{\max }^{+}$) as a function of the applied magnetic field are shown on Fig. 3(a). Note that the uniaxial symmetry case is unique for two reasons. First, the magnetization angular positions $\theta_{\min }^{-}$and $\theta_{\min }^{+}$corresponding to the two energy minima do not evolve with field. For $H<-H_{\mathrm{sw}}^{0}\left(H>H_{\mathrm{sw}}^{0}\right)$ the magnetization angular position $\theta_{\max }^{-}\left(\theta_{\max }^{+}\right)$corresponding to the two energy maxima do not evolve with field either. In those cases, $\theta_{\min }^{+}=\theta_{\max }^{-}=0^{\circ}$, which corresponds to the parallel $(\mathrm{P})$ alignment of the spin- valve, and $\theta_{\min }^{-}=\theta_{\max }^{+}=180^{\circ}$, which corresponds to its antiparallel (AP) alignment. Second, as an energy minimum vanishes it is replace by an energy maximum at the same position. Consequently, if the system moves from a minimum to a maximum energy state, $E_{\min }^{-}$to $E_{\max }^{+}$at 

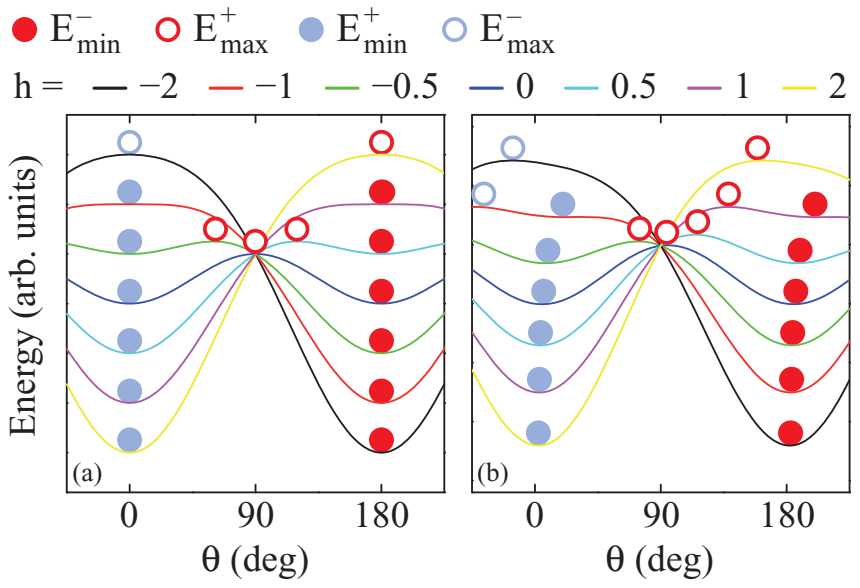

FIG. 2. (Color online) Evolution of the energy landscape of a macrospin system as a function of the angle $\theta$ between the perpendicular axis and the macrospin for different reduced applied magnetic fields $\left(h=\frac{H}{H_{\mathrm{k}}}\right)$ (a) with the uniaxial symmetry $\left(\lambda=0^{\circ}\right)$ and (b) when it has been broken $\left(\lambda=5^{\circ}\right)$. The circles highlight the evolution of the equilibrium positions and permit to define the two energy minima $E_{\min }^{-}$and $E_{\min }^{+}$and the two energy maxima $E_{\max }^{-}$and $E_{\max }^{+}$.

$H=H_{\mathrm{sw}}^{0}$ or $E_{\mathrm{min}}^{+}$to $E_{\mathrm{max}}^{-}$at $H=-H_{\mathrm{sw}}^{0}$, no change in the magnetization orientation is noticeable.

Let us focus on the AP-to-P switching which corresponds to one line of the current versus field state diagram. The system is initially set in the $E_{\text {min }}^{-}$energy state. From the power calculation one can tell for which set of field and current the magnetization shall switch. Figure 3(c) shows the evolution of the power received by the free layer in the AP magnetic configuration as a function of the applied magnetic field for different injected currents. Each curve is a concave parabola. At zero current, the power is always negative so the switching occurs when the $E_{\text {min }}^{-}$energetic state disappears at $H=H_{\mathrm{sw}}^{0}$. Indeed, the system has to stay in an energy minimum. Note that at the reversal, the power tends to zero. Consequently, as soon as a current is injected, the power becomes positive over a certain field range. Any injected current will therefore affect the switching magnetic fields. If the injected current is positive, the power becomes positive for $H<H_{\mathrm{sw}}^{0}$ so the system switches from the energy minimum $E_{\min }^{-}$to one of the two other available energy states ( $E_{\mathrm{max}}^{-}$if $H<-H_{\mathrm{sw}}^{0}$ or $E_{\mathrm{min}}^{+}$if $H>-H_{\mathrm{sw}}^{0}$ ), i.e., to the $\mathrm{P}$ magnetic configuration. The reversal occurs for a lower field than in the no-current case. On the contrary, if the injected current is negative, the power changes sign first at $H=H_{\mathrm{sw}}^{0}$. At this point a $E_{\min }^{-}$to $E_{\max }^{+}$transition is taking place, but since it corresponds to the same magnetization orientation no switching is observed $\left[\theta_{\min }^{-}\left(H_{\mathrm{sw}}^{0}\right)=\theta_{\mathrm{max}}^{+}\left(H_{\mathrm{sw}}^{0}\right)\right]$. The reversal occurs for larger fields $\left(H>H_{\mathrm{sw}}^{0}\right)$ when the power becomes negative again. The magnetization then switches into the $E_{\min }^{+}$ energy state, i.e., to the $\mathrm{P}$ magnetic configuration. The inset of Fig. 4 presents the state diagram calculated using this method. The linear evolution of the switching fields with the injected current is clearly visible as expected. Note that solving the equation $P_{\mathrm{t}}=0$ with $\lambda=0$ one can obtain the analytical expression of the switching current $I_{\mathrm{sw}}=\left(\frac{\alpha \mu_{0} \gamma}{\beta}\right) H \pm I_{\mathrm{sw}}^{0}$ with
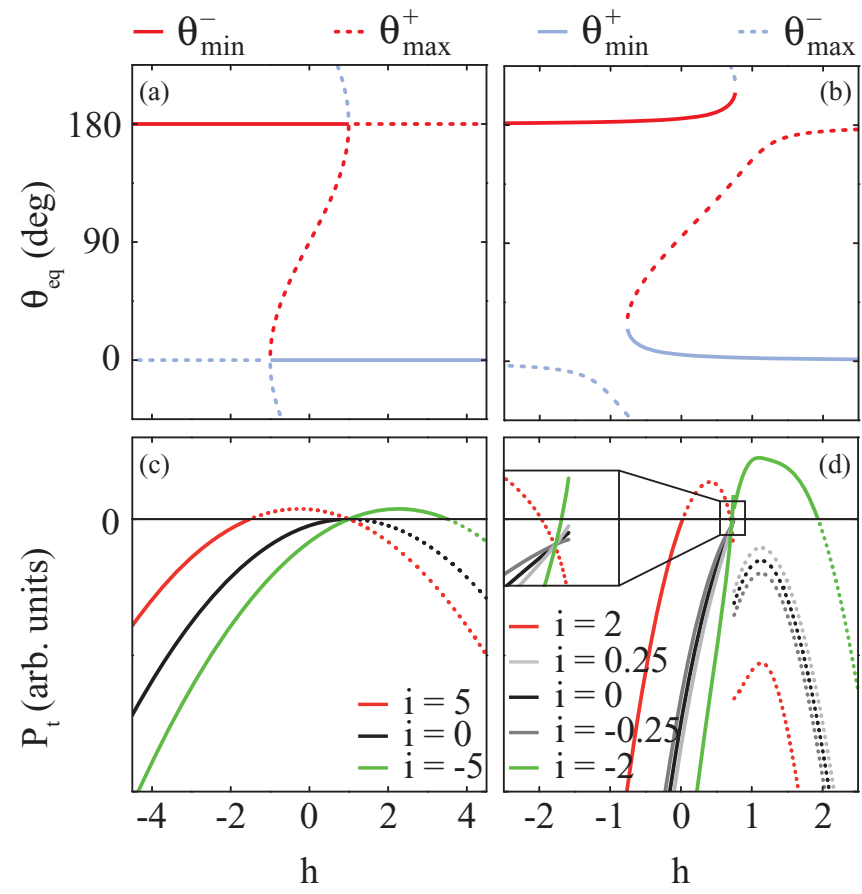

FIG. 3. (Color online) Equilibrium angular positions of the freelayer magnetization as a function of the reduced applied magnetic field $\left(h=\frac{H}{H_{\mathrm{k}}}\right)$ (a) with the uniaxial symmetry $\left(\lambda=0^{\circ}\right)$ and (b) when it has been broken $\left(\lambda=5^{\circ}\right)$. Total power received by the system as a function of the reduced applied field in the $E_{\min }^{-}$state when $H<$ $H_{\mathrm{sw}}^{0}$ and in the $E_{\max }^{+}$state when $H>H_{\mathrm{sw}}^{0}$, i.e., in the AP magnetic configuration for different reduced injected current $\left(i=\frac{I}{I_{\mathrm{sw}}^{0}}\right)$ (c) with the uniaxial symmetry $\left(\lambda=0^{\circ}\right)$ and (d) when it has been broken $\left(\lambda=5^{\circ}\right)$. Full and dashed lines respectively correspond to the power before and after the magnetization reversal.

$I_{\mathrm{sw}}^{0}=\left(\frac{\alpha \mu_{0} \gamma}{\beta}\right) H_{\mathrm{k}}$, which is exactly what has been previously calculated by a stability analysis of the LLGS equation. ${ }^{11}$

Let us now focus on the case where the uniaxial symmetry is broken $(\lambda \neq 0)$. All the calculation were performed with $\lambda=5^{\circ}$. Figure 2 (b) shows the evolution of the magnetic energy of the free layer as a function of $\theta$ for different applied magnetic fields. The field at which the energy minima vanishes is $H_{\mathrm{sw}}^{0} \neq H_{\mathrm{k}}$. Figure 3(b) shows the evolution of the equilibrium positions as a function of the applied magnetic field. The description of these equilibrium positions is similar to the one in the previous case. However, the system never reaches exactly the $\mathrm{AP}$ and $\mathrm{P}$ magnetic configurations because the equilibrium positions are never at fixed positions and always different from $\theta=0^{\circ}$ or $\theta=180^{\circ}$. Moreover, one can see that when an energy minimum vanishes at $H=H_{\mathrm{sw}}^{0}$ and at $H=-H_{\mathrm{sw}}^{0}$ the orientation of the magnetization has to change abruptly since no equilibrium state exists with the same magnetic orientation $\left[\theta_{\text {min }}^{-}\left(H_{\mathrm{sw}}^{0}\right) \neq \theta_{\max }^{+}\left(H_{\mathrm{sw}}^{0}\right)\right.$ and $\left.\theta_{\text {min }}^{+}\left(-H_{\mathrm{sw}}^{0}\right) \neq \theta_{\max }^{-}\left(-H_{\mathrm{sw}}^{0}\right)\right]$.

Again, let us analyze the reversal occurring starting with an initial energy state being $E_{\min }^{-}$. Figure $3(\mathrm{~d})$ presents the evolution of the power received by the free layer as a function of the applied magnetic field for different injected currents. Note that the power is calculated for a magnetization in the energy state $E_{\text {min }}^{-}$for $H<H_{\mathrm{sw}}^{0}$ and $E_{\mathrm{max}}^{+}$for $H>H_{\mathrm{sw}}^{0}$, i.e., a system always 


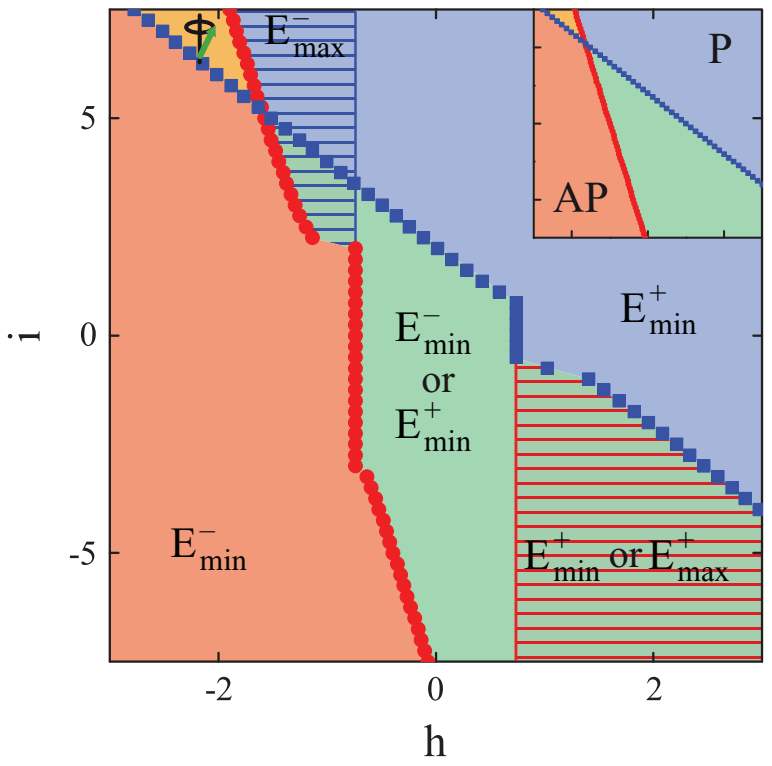

FIG. 4. (Color online) Calculated state diagram: Injected current as a function of the applied magnetic field using reduced coordinates ( $h=\frac{H}{H_{\mathrm{k}}}$ and $i=\frac{I}{I_{\mathrm{sw}}^{0}}$ ) when the uniaxial symmetry has been broken $\left(\lambda=5^{\circ}\right)$. The blue squares (red circles) mark the switching close to an AP-to-P (P-to-AP) reversal. In the red bottom left-hand (blue top right-hand) area the system is in the energy minimum $E_{\min }^{-}\left(E_{\min }^{+}\right)$. In the red bottom right-hand (blue top left-hand) dashed area the system is in the energy maximum $E_{\max }^{+}\left(E_{\max }^{-}\right)$. The red bottom right-hand (blue top left-hand) vertical line marks the $E_{\min }^{-}$to $E_{\max }^{+}\left(E_{\min }^{+}\right.$to $\left.E_{\max }^{-}\right)$ switching. Note that $E_{\min }^{-}\left(E_{\min }^{+}\right)$corresponds to a magnetic configuration close to an $\mathrm{AP}(\mathrm{P})$ state. $E_{\max }^{-}$and $E_{\max }^{+}$are canted states. The green bottom right-hand area corresponds to bistability regions. The yellow top left-hand area corresponds to the precession region. (Inset) Same state diagram calculated with the uniaxial symmetry $\left(\lambda=0^{\circ}\right)$.

close to the AP magnetic configuration. If the system is still in the $E_{\min }^{-}$energetic state at $\theta_{\min }^{-}$for $H=H_{\mathrm{sw}}^{0}$, a switching will take place. Either the magnetization orientation will become $\theta_{\max }^{+}$for the $E_{\max }^{+}$energy state with a system still close to the AP magnetic configuration or $\theta_{\text {min }}^{+}$for the $E_{\text {min }}^{+}$energy state with a system now close to the $\mathrm{P}$ magnetic configuration. Note that a magnetization oriented along $\theta_{\max }^{-}$or $\theta_{\max }^{+}$corresponds to what has been defined as a canted state in Ref. 12 .
At zero current, the power is always negative so the switching occurs in a similar fashion as the uniaxial case at $H=H_{\mathrm{sw}}^{0}$. The system switches from $E_{\min }^{-}$to $E_{\min }^{+}$which is close to an AP-to-P reversal. However, contrary to the previous case, the power is strictly negative and does not tend to zero. As a result, for moderate injected currents the power remains always negative and the switching still occurs at $H=H_{\mathrm{sw}}^{0}$. The spin-polarized current has therefore to exceed a critical value, for each polarity, to modify the switching fields. Above these thresholds, a positive current reduces the switching field and a negative current increases it as in the uniaxial case. Figure 4 presents the state diagram calculated in this case which exhibits regions around the zero current switching field where the switching fields are not affected by the injected current and regions where they evolve linearly with the current, as observed experimentally. ${ }^{12,13}$ It also shows where precessions and canted states may be observed. ${ }^{14}$

In summary, we have developed a general model to describe both current- and field-induced magnetization reversal. Indeed, starting from the Stoner-Wohlfarth energetic description, an analysis of the power dissipated or gained by the system provides a stability analysis permitting the prediction of magnetization reversal. The power is coming from the damping and the spin-transfer torques acting on the magnetization. This description also explains the appearance of others states only accessible when current is injected. This energy barrier analysis gives an intuitive understanding of the opening of the gaplike features of the switching phase boundary of nanopillar spin valves with perpendicular magnetizations. Indeed, the symmetry breaking generates the apparition of an intrinsic energy dissipation that the spin-polarized current has to overcome before affecting the switching fields. The cause of the symmetry breaking still needs to be investigated in detail. In conclusion, the power dissipation approach is complementary to the LLGS model, which gives access to magnetization trajectory. However, the proposed magnetization reversal description in terms of possible equilibrium state and energy barrier crossing is conceptually clearer and computationally simpler.

This work was supported in parts by the Friends contract of the French National Research Agency (ANR) and by the NSF. *stephane.mangin@ijl.nancy-universite.fr

${ }^{1}$ T. L. Gilbert, IEEE Trans. Magn. 40, 3443 (2004).

${ }^{2}$ E. C. Stoner and E. P. Wohlfarth, Proc. R. Soc. London A 240, 599 (1948).

${ }^{3}$ L. Berger, Phys. Rev. B 54, 9353 (1996).

${ }^{4}$ J. C. Slonczewski, J. Magn. Magn. Mater. 159, L1 (1996).

${ }^{5}$ M. D. Stiles and J. Miltat, Spin-Transfer Torque and Dynamics, Topics in Applied Physics, Vol. 101 (Springer, Berlin, 2006), pp. 225-308.

${ }^{6}$ T. Devolder, C. Chappert, J. A. Katine, M. J. Carey, and K. Ito, Phys. Rev. B 75, 064402 (2007).

${ }^{7}$ D. Bedau, H. Liu, J.-J Bouzaglou, A. D. Kent, J. Z. Sun, J. A. Katine, Eric E. Fullerton, and S. Mangin, Appl. Phys. Lett. 96, 022514 (2010); D. Bedau, H. Liu, J. Z. Sun, J. A. Katine, E. E. Fullerton, S. Mangin, and A. D. Kent, ibid. 97, 262502 (2010).
${ }^{8}$ B. Özyilmaz, A. D. Kent, D. Monsma, J. Z. Sun, M. J. Rooks, and R. H. Koch, Phys. Rev. Lett. 91, 067203 (2003).

${ }^{9}$ J. Grollier, V. Cros, H. Jaffres, A. Hamzić, J. M. Georges, G. Faini, J. Ben Youssef, H. Le Gall, and A. Fert, Phys. Rev. B 67, 174402 (2003).

${ }^{10}$ J. Z. Sun, Phys. Rev. B 62, 570 (2000).

${ }^{11}$ Y. B. Bazaliy, B. A. Jones, and S. C. Zhang, Phys. Rev. B 69, 094421 (2004).

${ }^{12}$ S. Mangin, D. Ravelosona, J. A. Katine, M. J. Carey, B. D. Terris, and E. E. Fullerton, Nat. Mater. 5, 210 (2006).

${ }^{13}$ S. Mangin, Y. Henry, D. Ravelosona, J. A. Katine, and E. E. Fullerton, Appl. Phys. Lett. 94, 012502 (2009).

${ }^{14}$ W. Lin, J. Cucchiara, C. Berthelot, T. Hauet, Y. Henry, J. A. Katine, E. E. Fullerton, and S. Mangin, Appl. Phys. Lett. 96, 252503 (2010). 